\title{
Evaluation of Quality of Life and Psychological Response in Recurrent and Metastatic Tumors Treated with Palliative Radiotherapy
}

\author{
Takafumi Yamano, Takeo Takahashi*, Shuichi Ueno, Yuki Iigima, Keiichiro Nishimura, \\ Kana Washizu, Rikana Soda, Nobuko Utsumi, Mikito Hondo, Munefumi Shimbo, \\ Shogo Hatanaka, Masatsugu Haryu
}

Department of Radiation Oncology, Saitama Medical Center, Saitama Medical University, Saitama, Japan

Email: *taketaka@saitama-med.ac.jp

How to cite this paper: Yamano, T., Takahashi, T., Ueno, S., Iigima, Y., Nishimura, K., Washizu, K., Soda, R., Utsumi, N., Hondo, M., Shimbo, M., Hatanaka, S. and Haryu, M. (2018) Evaluation of Quality of Life and Psychological Response in Recurrent and Metastatic Tumors Treated with Palliative Radiotherapy. Journal of Cancer Therapy, 9, 351-361.

https://doi.org/10.4236/jct.2018.94032

Received: March 16, 2018

Accepted: April 27, 2018

Published: April 30, 2018

Copyright (C) 2018 by authors and Scientific Research Publishing Inc. This work is licensed under the Creative Commons Attribution International License (CC BY 4.0).

http://creativecommons.org/licenses/by/4.0/

cc) (†) Open Access

\begin{abstract}
Objective: We evaluated the effects of palliative radiotherapy for cancer recurrence or metastasis on patient QOL and psychophysiology. Materials and Methods: Sixty seven patients who received palliative radiotherapy between 2014 and 2015 were enrolled. Patient diseases were bone metastasis in 51 patients, lymph node metastasis in 7 patients, brain metastasis in 2 patients, local recurrence in 3 patients, and others in 4 patients. Median irradiated dose was 30 Gy in 10 fractions for palliative radiotherapy. We used the questionnaires EORTC-QLQ-C30 and EORTC-QLQ-C15-PAL to evaluate patient QOL and the Hospital Anxiety and Depression Scale to evaluate patient mental healthcare at the start and at the end of radiotherapy. Results: As compared to scores at the start of radiotherapy, at the end of radiotherapy, NRS and face scale significantly decreased. On the other hand, Eastern Cooperative Oncology Group Performance Status did not show any changes during palliative radiotherapy. In functional scales, average scores of RF2 and EF also improved. In symptom scales, average scores of FA, PA, and SL improved. In bone metastasis group, global health status/QOL, PA, and SL significantly improved. There was relationship between anxiety improvement and QOL improvement after palliative radiotherapy. Nausea and vomiting scores of EORTC-QLQ-C15-PAL were associated with the irradiated volume of palliative radiotherapy for pelvic region. Conclusion: Patient QOL of was improved by palliative radiotherapy regardless of PS. The possibility of palliative radiotherapy having a positive influence on patient psychophysiology was also suggested in younger age.
\end{abstract}

\section{Keywords}

QOL, Palliative Radiotherapy, Psychological Response, Recurrent Tumor, Metastasis 


\section{Introduction}

Pain in cancer patients does not solely refer to physical pain, but various factors comprising the quality of life (QOL) are complexly involved. The goal of cancer treatment has been to prolong survival or improve local control. However, recent studies emphasized the importance of pain relief or QOL improvement. In particular, as the purpose of palliative therapy for recurrent or metastatic tumors, pain relief or QOL improvement is more important than survival prolongation or an improvement in local control. Palliative radiotherapy for recurrent or metastatic tumors plays an important role in symptom relief. In particular, the level of evidence regarding palliative radiotherapy for painful bone metastasis is high, and the pain improvement rating is reportedly $60 \%$ to $90 \%$ [1] [2]. In addition to cases of painful bone metastasis, palliative radiotherapy is indicated for cases in which the causal relationship between pain and tumors is clear, such as nervous tumor infiltration-related pain. The efficacy of much evidence regarding palliative radiotherapy was demonstrated by the pain improvement rating. For palliative care, it is important to evaluate subjective, patient-based outcomes. Currently, qualitative evaluation methods, such as the Numerical Rating Scale (NRS) and Face Scale, are commonly used as subjective outcome-evaluating methods. These methods facilitate simple investigation of the degree of pain, and are routinely selected. However, qualitative evaluation methods are not sufficient for cancer patients' QOL assessment. On the other hand, there are quantitative evaluation methods as methods of objectively evaluating each QOL item. In the field of oncology, disease-specific QOL scales include the European Organization for Research and Treatment Cancer (EORTC) QLQ-C30 [3] and Functional Assessment of Cancer Therapy (FACT) [4]. In Japan, few studies have adopted a QOL scale for patients treated by radiotherapy. No study has evaluated patients treated by palliative radiotherapy using an adequate, international QOL scale. In addition, no study has compared/discussed the field/volume of irradiation from the viewpoint of the adverse effects of palliative radiotherapy.

The purpose of this study was to clarify changes in the QOL and psychophysiological state before and after palliative radiotherapy for recurrent/metastatic tumors, which remain to be clarified in Japan, using adequate QOL scales, which are internationally/commonly used in cancer/palliative care patients.

\section{Materials and Methods}

Prior to this study, its protocol was approved by the Ethics Review Board of Saitama Medical Center, Saitama Medical University as a prospective clinical study (Approval No.: 997, 997-II, 1494). Sixty-seven patients with recurrent-/metastatic-tumor-related symptoms, such as pain/stress, between October 2014 and January 2016, from whom informed consent was obtained, were enrolled. Radiation oncologists evaluated the performance status (PS), NRS, and Face Scale scores by inquiry within 2 days before the start of radiotherapy and at its completion. The subjects were instructed to write a survey sheet on the QOL 
and psychophysiological state within 2 days before the start of radiotherapy and at its completion. On the same day, the sheet was collected.

The following items were investigated before the start of palliative radiotherapy (within 2 days) and at its completion. The general condition was evaluated using the Eastern Cooperative Oncology Group (ECOG) Performance Status (PS). Qualitative evaluation methods were assessed using the NRS and Face Scale. Quantitative evaluation methods were evaluated using the EORTC QLQC30 [3] [5] and EORTC QLQ-C15-PAL [6] with respect to the QOL, as well as the Hospital Anxiety and Depression Scale (HADS) [7] with respect to the psychophysiological state. The HADS involves the scoring of "anxiety" and "depression". For statistical analysis, the PS was compared using Fisher's exact test. The EORTC QLQ-C30, EORTC QLQ-C15-PAL, and HADS scores in each domain were compared before and after radiotherapy using Wilcoxon signedrank test.

\section{Results}

Patient diseases were bone metastasis in 51 patients, lymph node metastasis in 7 patients, brain metastasis in 2 patients, local recurrence in 3 patients, and others in 4 patients (Table 1). Median irradiated dose was 30 Gy in 10 fractions for palliative radiotherapy. The median age of the subjects was 67.5 years (range: 37 88 years). They consisted of 46 males and 21 females. With respect to the primary focus, lung cancer was the most frequent $(n=27)$. Concerning the mode of metastasis/relapse, bone metastasis was the most frequent $(n=51)$, accounting for $76.1 \%$. Of the 67 patients, 49 received oral opioids during the irradiation period (Table 1). No patient received chemotherapy or treatment with molecule-targeting drugs during palliative radiotherapy.

Of the 67 patients, palliative radiotherapy was accomplished in 63 (94\%).

Of the 63 patients, the results of assessment with the EORTC QLQ-C30 and EORTC QLQ-C15-PAL before and after radiotherapy were analyzed in 61, excluding 2 from whom survey sheets could not be collected (EORTC QLQ-C30 and EORTC QLQ-C15-PAL collection rates: 91\%). Concerning response defects in the survey sheets collected, the score of each domain was calculated using corrected values according to a scoring manual.

The HADS scores before and after radiotherapy were analyzed in 54 patients (collection rate: $80.6 \%$ ).

PS: Before radiotherapy, 28 and 34 patients showed favorable and unfavorable PS scores, respectively (PS 0 - 1 and PS 2 - 3 groups, respectively). After radiotherapy, the two groups consisted of 29 patients each (Table 2). There were no significant changes in the PS after palliative radiotherapy.

Palliative radiotherapy decreased the number of patients with an unfavorable NRS score (score: 7 - 10) from 22 to 3, showing an improvement. It also decreased the number of patients with an unfavorable Face Scale score (score: 4 - 5) from 23 to 2, showing an improvement (Table 2). Overall, the pain improvement 
Table 1. Patient characteristics and prescribed dose of palliative radiotherapy.

\begin{tabular}{|c|c|c|c|c|c|}
\hline age & & metastasis & & Histopathology & \\
\hline$\geq 70$ & 31 & bone & 51 & adenocarcinoma & 35 \\
\hline$<70$ & 36 & lymph node & 7 & $\begin{array}{l}\text { squamous cell } \\
\text { carsinoma }\end{array}$ & 9 \\
\hline median & $67.5(37-88)$ & brain & 2 & urothelial carcinoma & 4 \\
\hline gender & & pleura & 2 & clear cell carcinoma & 4 \\
\hline male & 46 & muscle, skin & 2 & small cell carcinoma & 3 \\
\hline female & 21 & loal reccurence & 3 & others & 12 \\
\hline parimary site & & prescpription dose & & & \\
\hline lung & 27 & median & 30 Gy (20 - 54 Gy) & & \\
\hline prostate & 8 & $\geq 30 \mathrm{~Gy}$ & 56 & & \\
\hline kidny & 5 & $<30 \mathrm{~Gy}$ & 11 & & \\
\hline bladder & 5 & opioids & & & \\
\hline colon, rectum & 4 & yes & 49 & & \\
\hline esophagus & 3 & no & 18 & & \\
\hline pancreas & 3 & & & & \\
\hline others & 7 & & & & \\
\hline
\end{tabular}

Table 2. PS, NRS, and face scale before and after treatment of palliative radiotherapy.

\begin{tabular}{ccc}
\hline PS & before RT & after RT \\
\hline $0-1$ & 28 & 29 \\
$2-3$ & 34 & 29 \\
4 & 1 & 0 \\
NRS & before RT & after RT \\
$0-3$ & 10 & 40 \\
$4-6$ & 27 & 14 \\
$7-10$ & 22 & 3 \\
Face Scale & before RT & after RT \\
$0-1$ & 2 & 14 \\
$2-3$ & 34 & 2 \\
$4-5$ & 23 & 39 \\
\hline
\end{tabular}

RT: Radiotherapy. PS: Performance Status. NRS: Nemerical Rating Scale.

rating (NRS improvement rating) was $81.8 \%$ (45/55). In patients with bone metastasis (bone metastasis group), it was $84.4 \%$ (38/45).

When analyzing the results of assessment with the EORTC QLQ-C30, there 
were significant improvements in the "pain" and "insomnia" scores. On the other hand, "nausea/vomiting" and "diarrhea" significantly exacerbated (Table 3). When analyzing the EORTC QLQ-C15-PAL results, there were significant improvements in the "emotional function", "fatigue", and "pain" scores. On the other hand, "nausea/vomiting" significantly exacerbated (Table 4). In patients aged $<70$ years, both the EORTC QLQ-C30 and EORTC QLQ-C15-PAL results showed a significant improvement in the total QOL score.

In the bone metastasis group, the EORTC QLQ-C30 results showed an improvement in the total QOL score in addition to improvements in the "pain" and "insomnia" scores, which significantly improved overall (Table 5). The EORTC QLQ-C15-PAL results showed significant improvements in the "emotional function", "fatigue", "pain", and "insomnia" scores. On the other hand, "nausea/vomiting" significantly exacerbated (Table 6).

On assessment with the HADS, there were no significant changes in the "anxiety" or "depression" scores after palliative radiotherapyin comparison with the pre-treatment values. However, there was an improvement in the "anxiety" score in patients aged $\leq 70$ years, and it was correlated with symptom relief.

Table 3. Scores of EORTC QLQ-C30 before and after palliative radiotherapy for all patients.

\begin{tabular}{|c|c|c|c|}
\hline \multicolumn{4}{|c|}{ Results of EORTC QLQ-C30 about all Patients } \\
\hline domain & before RT & after RT & $p$ value \\
\hline QL2 & $34.5 \pm 22.8$ & $42.5 \pm 23.2$ & 0.07 \\
\hline PF2 & $61.8 \pm 26.5$ & $61.2 \pm 26.1$ & 0.84 \\
\hline RF2 & $40.6 \pm 32.4$ & $46.1 \pm 32.7$ & 0.16 \\
\hline $\mathrm{EF}$ & $62.4 \pm 24.3$ & $65.2 \pm 26.0$ & 0.16 \\
\hline $\mathrm{CF}$ & $66.4 \pm 23.7$ & $63.5 \pm 24.7$ & 0.24 \\
\hline SF & $65.5 \pm 29.5$ & $57.2 \pm 31.1$ & 0.11 \\
\hline FA & $53.5 \pm 25.7$ & $50.4 \pm 25.0$ & 0.47 \\
\hline NV & $10.6 \pm 18.2$ & $16.7 \pm 22.7$ & 0.02 \\
\hline PA & $66.9 \pm 28.0$ & $51.4 \pm 26.8$ & $<0.01$ \\
\hline DY & $32.2 \pm 29.8$ & $32.2 \pm 31.0$ & 0.75 \\
\hline SL & $52.3 \pm 29.1$ & $39.7 \pm 29.3$ & $<0.01$ \\
\hline $\mathrm{AP}$ & $41.2 \pm 33.8$ & $47.5 \pm 33.2$ & 0.09 \\
\hline $\mathrm{CO}$ & $35.6 \pm 32.7$ & $36.1 \pm 32.9$ & 0.72 \\
\hline DI & $10.3 \pm 21.6$ & $19.5 \pm 29.1$ & 0.01 \\
\hline FI & $35.0 \pm 30.9$ & $37.9 \pm 33.3$ & 0.71 \\
\hline
\end{tabular}

QL2: Global Health Status. PF2: Physical Function. RF2: Role Function. EF: Emotional Function. CF: Cognitive Function. SF: Social Function. FA: Fatigue. NV: Nausea Vomiting. PA: Pain. DY: Dyspnea. SL: Insomnia. AP: Appetite Loss. CO: Constipation. DI: Diarrhea. FI: Financial Impact. RT: Radiotherapy. 
Table 4. Scores of EORTC QLQ-C15-PAL before and after palliative radiotherapy for all patients.

\begin{tabular}{cccc}
\hline \multicolumn{3}{c}{ Results of EORTC QLQ-C15PAL about all Patients } \\
\hline domain & before RT & after RT & $p$ value \\
QL2 & $36.8 \pm 24.7$ & $42.7 \pm 23.6$ & 0.19 \\
PF2 & $55.4 \pm 28.1$ & $58.1 \pm 28.1$ & 0.37 \\
EF & $\mathbf{5 7 . 6} \pm 31.2$ & $\mathbf{6 4 . 1} \pm \mathbf{2 7 . 2}$ & $\mathbf{0 . 0 4}$ \\
FA & $\mathbf{5 8 . 2} \pm \mathbf{2 8 . 8}$ & $\mathbf{4 9 . 9} \pm 24.1$ & $\mathbf{0 . 0 4}$ \\
NV & $\mathbf{8 . 9} \pm 20.3$ & $\mathbf{1 4 . 7} \pm 24.6$ & $\mathbf{0 . 0 3}$ \\
PA & $\mathbf{6 4 . 7} \pm 27.9$ & $\mathbf{5 0 . 0} \pm 27.2$ & $<0.01$ \\
DY & $25.3 \pm 25.0$ & $24.1 \pm 26.1$ & 0.6 \\
SL & $49.7 \pm 30.9$ & $38.4 \pm 29.3$ & 0.07 \\
AP & $40.2 \pm 32.6$ & $46.0 \pm 31.5$ & 0.07 \\
CO & $31.6 \pm 32.7$ & $33.9 \pm 33.3$ & 0.79 \\
\hline
\end{tabular}

QL2: Global Health Status. PF2: Physical Function. EF: Emotional Function. FA: Fatigue. NV: Nausea Vomiting. PA: Pain. DY: Dyspnea. SL: Insomnia. AP: Appetite Loss. CO: Constipation. RT: Radiotherapy.

Table 5. Scores of EORTC QLQ-C30 before and after palliative radiotherapy for patients with bone metastasis.

\begin{tabular}{cccc}
\hline domain & before RT & after RT & $p$ value \\
\hline QL2 & $30.0 \pm 21.6$ & $41.3 \pm 23.6$ & 0.02 \\
PF2 & $59.9 \pm 27.4$ & $59.1 \pm 25.5$ & 0.81 \\
RF2 & $36.2 \pm 30.8$ & $44.0 \pm 32.9$ & 0.1 \\
EF & $59.6 \pm 23.8$ & $63.3 \pm 27.6$ & 0.09 \\
CF & $64.4 \pm 24.3$ & $63.3 \pm 27.0$ & 0.67 \\
SF & $63.0 \pm 31.6$ & $55.2 \pm 31.8$ & 0.29 \\
FA & $55.6 \pm 26.4$ & $50.6 \pm 25.5$ & 0.23 \\
NV & $10.7 \pm 19.6$ & $15.9 \pm 23.8$ & 0.07 \\
PA & $72.7 \pm 24.9$ & $52.5 \pm 26.8$ & $<0.01$ \\
DY & $31.9 \pm 31.5$ & $32.6 \pm 28.8$ & 0.74 \\
SL & $55.1 \pm 28.8$ & $37.7 \pm 29.2$ & $<0.01$ \\
AP & $45.4 \pm 34.7$ & $47.5 \pm 34.9$ & 0.48 \\
CO & $35.5 \pm 33.3$ & $36.2 \pm 33.6$ & 0.92 \\
DI & $9.6 \pm 21.8$ & $17.0 \pm 25.9$ & 0.08 \\
FI & $37.0 \pm 33.5$ & $42.8 \pm 35.2$ & 0.41 \\
\hline
\end{tabular}

QL2: Global Health Status. PF2: Physical Function. RF2: Role Function. EF: Emotional Function. CF: Cognitive Function. SF: Social Function. FA: Fatigue. NV: Nausea Vomiting. PA: Pain. DY: Dyspnea. SL: Insomnia. AP: Appetite Loss. CO: Constipation. DI: Diarrhea. FI: Financial Impact. RT: Radiotherapy. 
Table 6. Scores of EORTC QLQ-C15-PAL before and after palliative radiotherapy for patients with bone metastasis.

\begin{tabular}{cccc}
\hline domain & before RT & after RT & $p$ value \\
\hline QL2 & $33.7 \pm 25.0$ & $41.3 \pm 24.0$ & 0.17 \\
PF2 & $53.8 \pm 27.8$ & $58.6 \pm 28.3$ & 0.49 \\
EF & $54.2 \pm 30.6$ & $62.7 \pm 29.2$ & 0.02 \\
FA & $61.9 \pm 28.5$ & $50.8 \pm 25.0$ & 0.02 \\
NV & $\mathbf{7 . 4} \pm 18.1$ & $\mathbf{1 4 . 1} \pm 25.8$ & $\mathbf{0 . 0 2}$ \\
PA & $\mathbf{6 9 . 5} \pm 25.3$ & $\mathbf{5 0 . 7} \pm 28.1$ & $<0.01$ \\
DY & $23.7 \pm 25.0$ & $25.2 \pm 25.5$ & 0.81 \\
SL & $\mathbf{5 1 . 4} \pm \mathbf{3 0 . 9}$ & $37.7 \pm 30.0$ & $\mathbf{0 . 0 2}$ \\
AP & $43.5 \pm 32.5$ & $45.7 \pm 32.9$ & 0.41 \\
CO & $32.6 \pm 33.0$ & $34.1 \pm 33.7$ & 0.85 \\
\hline
\end{tabular}

QL2: Global Health Status. PF2: Physical Function. EF: Emotional Function. FA: Fatigue. NV: Nausea Vomiting. PA: Pain. DY: Dyspnea. SL: Insomnia. AP: Appetite Loss. CO: Constipation. RT: Radiotherapy.

We examined "nausea/vomiting" and "diarrhea", of which the significant exacerbation was noted on assessment with the EORTC QLQ-C30 and EORTC QLQ-C15-PAL, with respect to the field of irradiation. These items showed significant exacerbation in patients who underwent pelvic irradiation (Figure 1). On pelvic irradiation, the irradiation volume was larger than in the other fields. When comparing the results between irradiation volumes of $\geq 1250 \mathrm{~mL}$ and $<1250 \mathrm{~mL}$ at $80 \%$ of the prescribed dose, "nausea/vomiting" and "diarrhea" in the EORTC QLQ-C30, as well as "nausea/vomiting" in the EORTC QLQ-C15-PAL, exacerbated in patients with an irradiation volume of $\geq 1250 \mathrm{~mL}$. In particular, the "nausea/vomiting" score in the EORTC QLQ-C15-PAL significantly increased (Figure 2).

\section{Discussion}

It is important to evaluate the QOL before and after palliative radiotherapy for recurrent or metastatic tumors [8], but no study has reported assessment using an adequate QOL scale in patients undergoing palliative radiotherapy in Japan. We previously conducted a survey involving patients undergoing radiotherapy using the QOL-ACD, and reported that there was a significant improvement in the QOL score during/after radiotherapy, and that there was a correlation between the QOL score and psychophysiological state [9]. However, the QOL-ACD did not involve any question item on pain [10]. In this study, we examined changes in the QOL after palliative radiotherapy for recurrent or metastatic tumors, which had not been clarified in Japan, using international QOL scales (EORTC QLQ-C30 and EORTC QLQ-C15-PAL), and indicated that improvements in the "pain", "insomnia", "emotional function", and "fatigue" scores were achieved. Caissie et al. conducted a survey involving patients with 

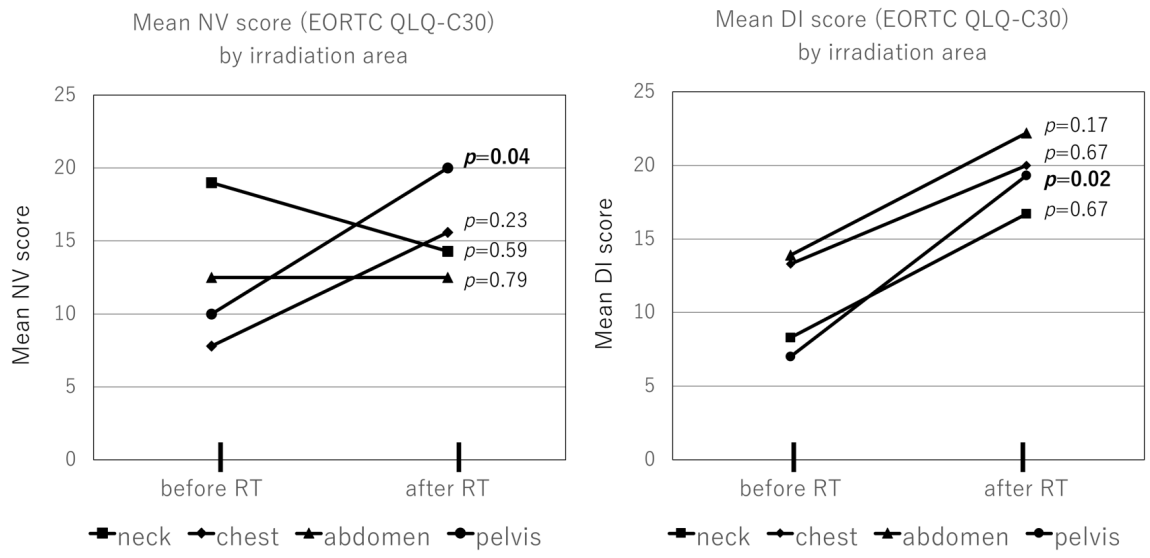

Figure 1. Mean nausea and vomiting score and diarrhea score before and after palliative radiotherapy in the irradiated field.

Mean score of DI, NV and NV15 by irradiation volume

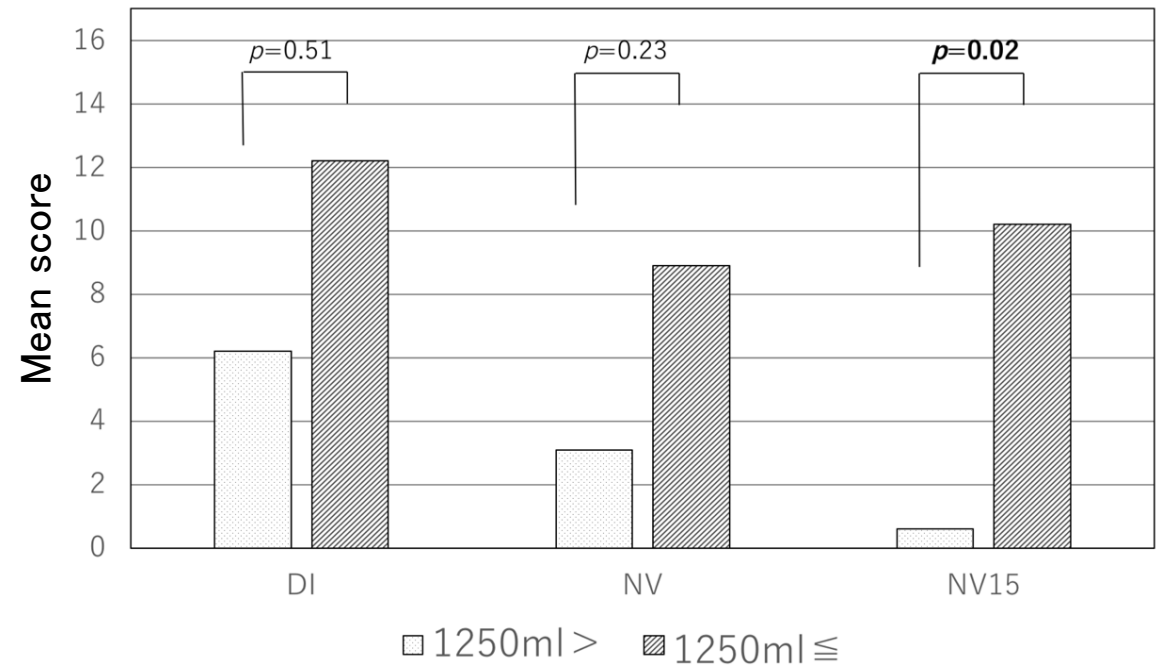

Figure 2. Mean score of DI, NV, NV15 in the irradiated field.

bone metastasis using the EORTC QLQ-C15-PAL for 2 months after radiotherapy, and reported that there were significant improvements in the "pain" and "insomnia" scores in all phases after radiotherapy in the pain relief group, with significant improvements in the "emotional function", "fatigue", and "global QOL" scores [11]. Lorenzo et al. evaluated the QOL using the EORTC QLQ-C30 in patients with bone metastasis from prostate cancer who underwent palliative radiotherapy, and indicated improvements in the "pain", "insomnia", and "fatigue" scores after palliative radiotherapy [12]. This study also showed significant improvements in similar items. According to the literature, several studies (including a study published by Caissie et al.) compared changes in the QOL between responders to irradiation (pain relief group) and non-responders (non-pain-relief group) among patients with bone metastasis [11] [13]. McDonald et al. conducted a systematic review of the QOL after palliative radiotherapy for bone metastasis, and reported that there was a marked improvement in the QOL in responders 
with pain relief [13]. These studies suggest the association between symptom relief and an improvement in the QOL after palliative radiotherapy in patients with bone metastasis. In this study, there was also a significant improvement in the "global QOL" score on assessment with the EORTC QLQ-C30 in the bone metastasis group with more marked pain than in the other patients.

Although a few studies investigated the relationship between the PS and QOL in patients undergoing radiotherapy, few studies have examined changes in the PS before and after palliative radiotherapy [14] [15]. The results of this study showed an improvement in the QOL after irradiation, whereas there were no significant changes in the PS after palliative radiotherapy. This suggests that recurrent/metastatic tumor patients' subjective QOL improves even when there is no change in the PS after palliative radiotherapy.

Concerning the influence of radiotherapy on the psychophysiological state, a study reported that there was a correlation between QOL/symptom deterioration and anxiety/depression [16] [17]. However, no study has examined palliative radiotherapy. In this study, overall, there were no significant changes in anxiety or depression after palliative radiotherapy. For palliative radiotherapy, an irradiation field is established so that the development of adverse effects may be minimized. In this study, there was no grade 2 or higher (CTCAE v4.0) adverse event in any subject, but "nausea/vomiting" and "diarrhea" significantly exacerbated after pelvic irradiation. In most patients undergoing pelvic irradiation, the lesion extent is more extensive than in thoracic or lumbar vertebral metastasis patients undergoing palliative radiotherapy; the irradiation volume tends to increase. In this study, there was no significant difference in the "nausea/vomiting" score at the completion of palliative radiotherapy between opioid-treated and non-opioid-treated patients. Therefore, the deterioration of "nausea/vomiting" after palliative radiotherapy may depend on the irradiation volume. In the future, it may be necessary to consider radiation doses to be prescribed, involving irradiation-field setting, for patients in whom the irradiation volume may increase, such as those with multiple or pelvic bone metastases.

\section{Conclusion}

Palliative radiotherapy markedly improves recurrent/metastatic tumor patients' QOL. In particular, a marked improvement was achieved in bone metastasis patients complaining of symptoms. The results suggest that the QOL, as a subjective outcome, improves even when there are no changes in the PS after palliative radiotherapy. Even for palliative radiotherapy in which an irradiation field/dose is established so that no adverse event may appear, an irradiation field should be carefully established from the viewpoint of adverse reactions/QOL when the irradiation volume increases.

\section{References}

[1] Chow, E., Zeng, L., Salvo, N., Dennis, K., Tsao, M. and Lutz, S. (2012) Update on the Systematic Review of Palliative Radiotherapy Trials for Bone Metastases. Clini- 
cal Oncology, 24, 112-124. https://doi.org/10.1016/j.clon.2011.11.004

[2] Lutz, S., Berk, L., Chang, E., Chow, E., Hahn, C., Hoskin, P., et al. (2011) Palliative Radiotherapy for Bone Metastases: An ASTRO Evidence-Based Guideline. International Journal of Radiation Oncology * Biology * Physics, 79, 965-976. https://doi.org/10.1016/j.ijrobp.2010.11.026

[3] Aaronson, N.K., Ahmedzai, S., Bergman, B., Bullinger, M., Cull, A., Duez, N.J., et al. (1993) The European Organization for Research and Treatment of Cancer QLQ-C30: A Quality of Life Instrument for Use in International Clinical Trials in Oncology. Journal of the National Cancer Institute, 85, 365-376. https://doi.org/10.1093/jnci/85.5.365

[4] Cella, D.F., Tulsky, D.S., Gray, G., Sarafian, B., Linn, E., Bonomi, A., et al. (1993) The Functional Assessment of Cancer Therapy Scale: Development and Validation of the General Measure. Journal of Clinical Oncology, 11, 570-579. https://doi.org/10.1200/JCO.1993.11.3.570

[5] Kobayashi, K., Takeda, F., Teramukai, S., Gotoh, I., Sakai, H., Yoneda, S., et al. (1998) A Cross-Validation of the European Organization for Research and Treatment of Cancer QLQ-C30 (EORTC QLQ-C30) for Japanese with Lung Cancer. European Journal of Cancer, 34, 810-815. https://doi.org/10.1016/S0959-8049(97)00395-X

[6] Groenvold, M., Morten, Aa., Petersen, M.A., Aaronson, N.K., Arraras, J.I., Blazeby, J.M., Bottomley, A., et al. (2006) The Development of the EORTC QLQ-C-15-PAL: A Shortened Questionnaire for Cancer Patients in Palliative Care. European Journal of Cancer, 42, 55-64. https://doi.org/10.1016/j.ejca.2005.06.022

[7] Zigmoid, A.S. and Snaith, R.P. (1982) The Hospital Anxiety and Depression Scale. Acta Psychiatrica Scandinavica, 67, 361-370. https://doi.org/10.1111/j.1600-0447.1983.tb09716.x

[8] Westhoff, P.G., Verdam, M.G., Oort, F.J., Jpbsen, J.J., van Vulpen, M., Leer, J.W., et al. (2016) Course of Quality of Life after Radiation Therapy for Painful Bone Metastasis: A Detailed Analysis From the Dutch Bone Metastasis Study. International Journal of Radiation Oncology ${ }^{*}$ Biology ${ }^{*}$ Physics, 95, 1391-1398. https://doi.org/10.1016/j.ijrobp.2016.03.032

[9] Takahashi, T., Hondo, M., Nishimura, K., Kitani, A., Yamano, T., Yanagita, H., et al. (2008) Evaluation of Quality of Life and Psychological Response in Cancer Patients Treated with Radiotherapy. Radiation Medicine, 26, 396-401. https://doi.org/10.1007/s11604-008-0248-5

[10] Kurihara, M., Shimizu, H., Tsuboi, K., Kobayashi, K., Murakami, M., Eguchi, K., et al. (1999) Development of Quality of Life Questionaire in Japan: Quality of Life Assessment of cancer Patients Receiving Chemotherapy. Psycho-Oncology, 8, 353-363. https://doi.org/10.1002/(SICI)1099-1611(199907/08)8:4<355::AID-PON401>3.0.CO ;2-I

[11] Caissie, A., Zeng, L., Nguyen, J., Zhang, L., Jon, F., Dennis, K., et al. (2012) Assessment of Health-Related Quality of Life with the European Organization for Research and Treatment of Cancer QLQ-C15-PAL after Palliative Radiotherapy of Bone Metastases. Clinical Oncology, 24, 125-133. https://doi.org/10.1016/j.clon.2011.08.008

[12] Di Loreczo, G., Autorino, R., Ciardiello, F., Raben, D., Bianco, C., Troiani, T., et al. (2003) External Beam Radiotherapy in Bone Metastatic Prostate Cancer: Impact on Patient's Pain Relief and Quality of Life. Oncology Reports, 10, 399-404. https://doi.org/10.3892/or.10.2.399 
[13] McDonald, R., Chow, E., Rowbottom, L., Bedard, G., Lam, H., Wong, E., et al. (2015) Quality of Life after Palliative Radiotherapy in Bone Metastases: A Literature Review. Journal of Bone Oncology, 4, 24-31. https://doi.org/10.1016/j.jbo.2014.11.001

[14] Caissie, A., Culleton, S., Nguyen, J., Zhang, L., Zeng, L., Holden, L., et al. (2012) EORTC QLQ-C15-PAL Quality of Life Scores in Patients with Advanced Cancer Referred for Palliative Radiotherapy. Support Care Cancer, 20, 841-848.

https://doi.org/10.1007/s00520-011-1160-6

[15] Cheon, P.M., Pulenzas, N., Zhang, L., Mauti, E., Wong, E., Thavarajah, N., et al. (2015) Fatigue Scores in Patients Receiving Palliative Radiotherapy for Painful Bone Metastases. Support Care Cancer, 23, 2097-2103.

https://doi.org/10.1007/s00520-014-2561-0

[16] Mystakidou, K., Tsilika, E., Parpa, E., Gogou, P., Panagiotou, I., Vassiliou, I., et al. (2013) Relationship of General Self-Efficacy with Anxiety, Symptom Severity and Quality of Life in Cancer Patients before and after Radiotherapy Treatment. Psyco-Oncology, 22, 1089-1095. https://doi.org/10.1002/pon.3106

[17] Gogou, P., Tsilika, E., Parpa, E., Kouvaris, J., Damigos, D., Balafouta, M., et al. (2015) The Impact of Radiotherapy on Symptoms, Anxiety and QoL in Patients with Cancer. Anticancer Research, 35, 1771-1776. 